\title{
SEED SIZE AND SEED QUALITY EFFECTS ON SEEDDLING GROWTH OF BARLEY VARIETIES GROWN IN Fe AND Zn DEFICIENT CALCAREOUS SOIL
}

\author{
Sadullah N. Al-Niemi ${ }^{1} \quad$ Mohammed Sedqi S. Dohuki ${ }^{2}$
}

${ }^{1}$ Univ. of Mosul, College of Agric. and Forestry, Dept. of Soil and Water Sci., MOSUL, IRAQ.

${ }^{2}$ Univ. of Dohuk, College of Agric., Dept. of Soil Sci. and Water, DOHUK, IRAQ.

\begin{abstract}
Greenhouse experiment was carried out to study the effects of seed size and seed iron and zinc content on seedling growth of 15 promising and dominant varieties of barley plant in the North part of Iraq. Three groups of seed size with different seed quality ( $\mathrm{Fe}$ and $\mathrm{Zn}$ content) of each variety were sown in $\mathrm{Fe}$ and $\mathrm{Zn}$ deficient alkaline calcareous soil. After six weeks root and shoot dry weights of barley plants grew from different seed size and seed quality were compared. Seedlings derived from larger seed size and with high Fe and $\mathrm{Zn}$ content had greater root and shoot dry weights. Barley varieties El-Kheir, 1/1105,Amal and local black performed better seedling growth.
\end{abstract}

\section{INTRODUCTION}

Micronutrient content of seeds is important for the nutrition of plant seedling and this effect is most pronounced in micronutrient deficient soil (Rengel and Graham, 1995, Yilmaz et al., 1997). Seed size and seed micronutrient content roles on seedling growth under $\mathrm{Fe}$ and $\mathrm{Zn}$ deficient condition is rarely studied.In Iraq there is no such kind of studies. In this study, the objectives were to investigate the effects of seed size and its micronutrients ( $\mathrm{Fe}$ and $\mathrm{Zn}$ ) content on the early growth of 15 promising and dominant barley varieties grown in north part of Iraq, on calcareous soil.

\section{MATERIALS AND METHODS}

Greenhouse experiment was conducted to determine the effects of seed size and seed micronutrients $(\mathrm{Fe}$ and $\mathrm{Zn})$ content on the early growth of 15 different barley varieties (El-Kheir, Boraq, Ghallion, 1/1105, Haddhar-465, Amal, AlBawadi, Sameer, Local black, Biba-99, ACSAD-14, ACSAD-2, ACSAD-9, ACSAD-12 and Najim Al-deen). The seeds of the 15 promising and dominant varieties were collected from the agriculture research stations which located in Dohuk and Erbil governorates in north part of Iraq. Seeds of each variety were sieved and separated to three seed size groups \{retained on $4.0 \mathrm{~mm}$ mesh (group I), passed through a $4.0 \mathrm{~mm}$ mesh and retained on $3.0 \mathrm{~mm}$ mesh (group II), and passed through a $3.0 \mathrm{~mm}$ mesh and retained on $2.36 \mathrm{~mm}$ mesh (group III) \}. Iron and Zinc in seeds of each group size and of each barley variety were determined by using method described by Chapman and Part ( 1961).

Micronutrient ( $\mathrm{Fe}$ and $\mathrm{Zn}$ )-deficient calcareous silty clay soil with about $21 \%$ $\mathrm{CaCO}_{3}$ and $\mathrm{pH} 8.6$ (Table 1) was collected from the field experiment station at the

The research is a part of the Ph.D. thesis of the $2^{\text {nd }}$ researcher Received 19/10/2009 accepted 4/1/2010 
college of Agriculture, Dohuk University, Northern Iraq. PVC pots $(9.5 \mathrm{~cm}$ diameter $\times 11 \mathrm{~cm}$ length) were filled with $500 \mathrm{~g}$ air-dry soil. Eight seeds from each group size were sown in each pot, and distilled water was added in amounts sufficient to bring soil water content to $75 \%$ of soil field capacity. Soil moisture content was kept at $75 \%$ of field capacity during the period of the experiment by weighing the pots daily and adding by distilled water to obtain the original wet weight. After germination plants were thinned to 4 plants per pot, and after 45 days from sowing plants were harvested. At harvest time soil was washed off root under running tap water. Both roots and shoots were separated gently and weighed.

\section{RESULTS AND DISCUSSION}

Physical and chemical characteristics of soil :Various physiochemical properties of soil are presented in (Table 1). Soil was calcareous which contained more than $200 \mathrm{CaCO}_{3} \mathrm{gm} \mathrm{kg}^{-1}$ soil and characterized by very low organic matter content $15.5 \mathrm{gm} \mathrm{kg}^{-1}$ soil and had high $\mathrm{pH}$ value 8.6. Concentration of DTPA-extractable Fe in soil was $2.79 \mathrm{mg} \mathrm{kg}^{-1}$ soil which was less than the adequate amount of $\mathrm{Fe}$ in calcareous soils (4 $\mathrm{mg} \mathrm{kg}^{-1}$ soil) as stated by Soltanpour and Schwab (1977), and it was marginal with the critical level for calcareous soils $\left(2.5 \mathrm{mg} \mathrm{kg}^{-1}\right.$ soil) obtained by Sims and Johnson (1991), while the zinc concentration of the soil was $0.55 \mathrm{mg}$ $\mathrm{kg}^{-1}$ soil which was less than marginal amount of $\mathrm{Zn}$ in calcareous soils $(0.6-1.0 \mathrm{mg}$ $\mathrm{kg}^{-1}$ soil) as (Soltanpoure and Schwab, 1977) stated, and was very close to the critical level (0.5 mg kg-1soil) obtained by Sims and Johnson(1991). The soil texture silty clay with high fractions of both silt and clay and low fraction of sand.

Table (1): Physicochemical properties of top soil sample (0-30) $\mathrm{cm}$.

\begin{tabular}{|c|c|c|c|}
\hline $\mathrm{pH}$ & 8.6 & Sand $\left(\mathrm{g} \mathrm{kg}^{-1}\right)$ & 90.60 \\
\hline $\mathrm{CaCO}_{3}\left(\mathrm{gm} \mathrm{Kg}^{-1}\right.$ soil) & 201.0 & Silt $\left(\mathrm{g} \mathrm{kg}^{-1}\right)$ & 487.50 \\
\hline Organic matter $\left(\mathrm{gm} \mathrm{Kg}^{-1}\right.$ soil $)$ & 15.5 & Clay $\left(\mathrm{g} \mathrm{kg}^{-1}\right)$ & 421.90 \\
\hline Available Fe $\left(\mathrm{mg} \mathrm{Kg}^{-1}\right.$ soil $)$ & 2.79 & Soil texture & Silty clay \\
\hline Available $\mathrm{Zn}\left(\mathrm{mg} \mathrm{Kg}^{-1}\right.$ soil $)$ & 0.55 & Moisture at Field capacity (\%) & 31.68 \\
\hline
\end{tabular}

Seed Size and Seed micronutrients content:The effect of seed size was significant for seed micronutrients ( $\mathrm{Fe}$ and $\mathrm{Zn}$ ) content (Table 2). The large seed size had a bigger store of $\mathrm{Fe}$ and $\mathrm{Zn}$ nutrients. Seeds of the 15 barley varieties varied significantly (Table 2) in their Fe and Zn content and therefore, the seed Fe content was higher than that of Zn. Najim Al-deen and ACSAD-2 varieties showed the lowest seed Fe content, while Biba-99 and ACSAD-2 showed the lowest seed $\mathrm{Zn}$ content.

Root dry matter:Root dry matter production of the barley seedling was influenced by seed size and seed micronutrients content as well as was by barley varieties. Large seed size which contained highest amount of ( Fe and $\mathrm{Zn}$ ) significantly resulted in better root production (Table 3 ) than the other two seed size groups. Barley variety also had significant effect in root dry matter production, where AlBawadi, Amal and ACSAD-9 varieties had higher root dry matter as compared to other barley varieties.

Shoot dry matter:Seed size and seed micronutrients content effect on shoot dry matter production were very similar to the effect on root dry matter production 
(Table 3). Seedling derived from larger size which had a bigger store of micronutrients (Table 4) produced more shoot dry matter. The results also showed significant variation in shoot dry matter production under the effect of barley varieties. El-kheir variety had higher shoot dry matter production while Najim Aldeen produced significantly less shoot growth.

Table (2): Effects of barley variety and seed size group (I, II and III) on Fe and Zn seed content.

\begin{tabular}{|c|c|c|c|c|c|c|c|c|}
\hline \multirow[t]{2}{*}{ Barley varieties } & \multicolumn{3}{|c|}{$\left(\mu \mathrm{g} \mathrm{Fe} \mathrm{Kg^{-1 } )}\right.$} & \multicolumn{3}{|c|}{$\left(\mu \mathrm{gZn} \mathrm{Kg^{-1 } )}\right.$} & \multicolumn{2}{|c|}{$\begin{array}{c}\text { Average variety } \\
\text { nutrient content } \\
(\mu \mathrm{g} \mathrm{Kg})\end{array}$} \\
\hline & $\mathbf{I}$ & II & III & $\mathbf{I}$ & II & III & $\mathbf{F e}$ & Zn \\
\hline El-Kheir & 171.14 & $1 \leq r . Y V$ & ITY.Y. & $71 .$. & Tr.r4 & $00 . Y V$ & $1 \leqslant 0.04$ & Tr.Y. \\
\hline Buraq & $17 . .1 \%$ & 149.0 & 91.4. & $\mathrm{V \Lambda .} \cdot \mathrm{V}$ & $0 \leqslant .14$ & $\leq \vee .7$ & $1 \times 9.14$ & 09.94 \\
\hline Ghallion & $1 V V .1 T$ & 174.7. & IrY.rV & $11 .$. & $00.1 T$ & $01 . Y$. & 109.4 & $00 . V A$ \\
\hline $1 / 1105$ & 1ro.A. & $11 \varepsilon . Y V$ & $110 . \mathrm{V}$ & 07.14 & $01 . Y V$ & OY.rT & $1 Y 1.94$ & Or.Ys \\
\hline Haddhar-465 & ITr.rT & $11 \mathrm{~V} .1 \mathrm{~T}$ & IYY.Y. & 71.04 & OY.IT & $\leqslant V_{.} \cdot V$ & $1 Y 0.04$ & 00.91 \\
\hline Amal & $I \Lambda \varepsilon . Y V$ & IrT.IT & $1 \leq \varepsilon . \cdot V$ & $M . Y$. & $\varepsilon r .1 T$ & $\leqslant \Lambda . \cdot \vee$ & IOY.NY & $0 \cdot . \wedge$. \\
\hline Al-Bawadi & $1 \wedge \varepsilon .+\varepsilon$ & $1 . \varepsilon . Y V$ & $1.8 .1 T$ & 77.14 & $\varepsilon Y_{.} \leqslant \cdot$ & Or.IT & 141.94 & 04.07 \\
\hline Sameer & $18 \cdot .7$. & 1ro.r. & IIr.YV & 79.14 & $\$ 1.1 \%$ & $\leqslant 1 . \wedge$ & 107.14 & 29.79 \\
\hline Local black & $1 \leq 0 . Y V$ & $1 \times 7 . \leqslant V$ & IYY.Y. & $\Delta v . \cdot V$ & $\varepsilon \leqslant . \wedge$. & $\leqslant 4 . \cdot V$ & $1+1 . r 1$ & $\leq 9.41$ \\
\hline Biba-99 & $1 Y \Lambda . \leqslant V$ & $1.0 .1 \mathrm{~T}$ & 1.V.rr & $\varepsilon 7 . r 4$ & $\leqslant 0.7$. & rY.TV & $11 \% .7 \varepsilon$ & $\$ 1.04$ \\
\hline ACSAD-14 & IAY.r. & $1 \leq 9 . \mathrm{VV}$ & $1 \leq \Lambda . Y V$ & $10 . \mathrm{rV}$ & To.r. & $0 \leqslant . \cdot V$ & 109.19 & 71.01 \\
\hline ACSAD-2 & $1 \leq 1 . r \mu$ & $1 . \varepsilon .1 T$ & VI.1r & $\varepsilon \vee .$. & $\varepsilon \varepsilon . \cdot V$ & $\leqslant 1 . r$ & 1.0 .04 & $\leq \leqslant .99$ \\
\hline ACSAD-9 & $1 \leq V .7$ & $1150 . .$. & VY.VY & $\Lambda \Lambda . r V$ & $V V_{.} \cdot r$ & $09 . \cdots$ & $111 . \leqslant \varepsilon$ & $V Y . \wedge \varepsilon$ \\
\hline ACSAD-12 & $10 \Lambda . \mu r$ & $1+9 . \cdot v$ & $1 \leq \cdot . \cdot V$ & VY.TV & ov. . V & Tr.IT & $1 \leq 0 . \wedge r$ & 74.97 \\
\hline Najim Al-deen & $1 \mathrm{r \Lambda} \cdot \mathrm{V}$ & A०.r. & Tr.or & Y^.Y. & Tะ.Y. & $\leqslant$ Y.r. & 91.94 & $\theta \Lambda . Y$. \\
\hline $\begin{array}{c}\text { Variety } \times \begin{array}{c}\text { Seed size } \\
\text { effect }\end{array} \\
\text { Adj. LSD, } 0.05\end{array}$ & \multicolumn{3}{|c|}{1.11} & \multicolumn{3}{|c|}{$r 1.74$} & Ir.ru & 14.01 \\
\hline \multicolumn{7}{|c|}{ Seed size micronutrient content $\left(\mu \mathrm{g} \mathrm{Kg}{ }^{-1}\right)$} & & \\
\hline group & \multicolumn{3}{|c|}{ Fe } & \multicolumn{3}{|c|}{ Zn } & & \\
\hline $\mathbf{I}$ & \multicolumn{3}{|c|}{$100.1 \Lambda$} & \multicolumn{3}{|c|}{$7 \varepsilon .7 V$} & & \\
\hline II & \multicolumn{3}{|c|}{$1 \times 9.11$} & \multicolumn{3}{|c|}{ or.ra } & & \\
\hline III & \multicolumn{3}{|c|}{117.4} & \multicolumn{3}{|c|}{$\varepsilon \wedge .70$} & & \\
\hline $\begin{array}{l}\text { Seed size effect } \\
\text { Adj. LSD, 0.05 }\end{array}$ & \multicolumn{3}{|c|}{$0 . \leqslant V$} & \multicolumn{3}{|c|}{0.09} & & \\
\hline
\end{tabular}

The results demonstrated that seed size and seed micronutrients content can improve an early growth of root and shoot of seedling varieties growing in alkaline calcareous soil. These result are consistent with the finding of Rengel and Graham, (1995) Mousavi-Nik et al.(1997) were a more vigorous crop is established by seeds with a high density of nutrients, including micronutrients, and with the results of (Main and Nafziger, 1992) who stated that seed size is an important determinant of seedling vigour and early growth of cereals.

Root and shoot growth were decreased significantly in seedling grown from small seed size with low Fe and $\mathrm{Zn}$ content, and the reduction of root and shoot growth using seeds from group III, and group II were $29.24 \%$ and $15.38 \%$, respectively for the root growth as compared to the root growth using seeds from group I and $30.93 \%$ and $12.14 \%$, respectively for the shoot growth. This reduction in root and shoot growth was attributed to insufficient of micronutrients supply which in turn decrease the plant metabolism and functions (Marschner, 1995, Mengel and Kirkby. 2001).

Barley varieties El-kheir, 1/1105, Amal and local black generally gave higher seedling growth and performed better than other varieties. Seed size and seed 
micronutrients content are an important factors for screening a large number of barley varieties depending on root and shoot growth parameters.

Table (3): Effects of barley seed size group (I, II and III) on root dry weight of seedling.

\begin{tabular}{|c|c|c|c|c|c|c|}
\hline \multirow{2}{*}{ Barley varieties } & \multicolumn{3}{|c|}{ Root dry weight $\left(\mathrm{g} \operatorname{pot}^{-1}\right)$} & \multirow{2}{*}{$\begin{array}{c}\text { Variety } \times \text { seed size } \\
\text { effect } \\
\text { Adj. LSD, } 0.05 \\
\end{array}$} & \multirow{2}{*}{$\begin{array}{l}\text { root dry } \\
\text { weight } \\
\left(\mathrm{g} \mathrm{pot}^{-1}\right)\end{array}$} & \multirow{2}{*}{$\begin{array}{l}\text { Variety Adj. } \\
\text { LSD, } 0.05\end{array}$} \\
\hline & I & II & III & & & \\
\hline El-Kheir & $\because . r^{\prime}$ &. .189 & $.1 \cdot 7$ & \multirow{15}{*}{$\because \cdot \Sigma V$} & $.17 r$ & \multirow{15}{*}{$\because \cdot r V$} \\
\hline Buraq & $\because .1 \vee \leq$ & $\because .1 T \varepsilon$ &. $.1 Y V$ & & $.1 \leqslant 0$ & \\
\hline Ghallion &. .194 & .1149 & .1150 & & $.10 r$ & \\
\hline $1 / 1105$ &. .194 & $\because .19$ & .1150 & & .179 & \\
\hline Haddhar-465 & U.MYY &. .171 & $.1 \%$ & & $\because .1 \mathrm{VI}$ & \\
\hline Amal & $\cdot$. YYT & $\cdot .1 \wedge \mathrm{V}$ & .110 & & $\because .199$ & \\
\hline Al-Bawadi & 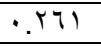 &. .191 & .179 & & $\because r \cdot \Lambda$ & \\
\hline Sameer & .17 &. .101 &. $.1 T r$ & & .10 & \\
\hline Local black & .190 & $\because .18$ & $.1 \leq r$ & & .179 & \\
\hline Biba-99 & .101 & $\because .10$ &. .111 & & $\because 1 \leq \varepsilon$ & \\
\hline ACSAD-14 & $\because .119$ & $\because 1 \leqslant r$ &.$I V Y$ & & .171 & \\
\hline ACSAD-2 &. .194 & $\because .1 \times 7$ & $.1 \cdot 1$ & & .109 & \\
\hline ACSAD-9 & $\cdot T^{\prime} \cdot$ & $\because .119$ &.. $\mathrm{VT}$ & & .191 & \\
\hline ACSAD-12 & $\cdot r \cdot \varepsilon$ & .179 & $\cdot 1 \cdot \varepsilon$ & & $\because .109$ & \\
\hline Najim Al-deen & $.1 \leq 7$ & $\because 1 \leq \varepsilon$ & $.1 \% \Lambda$ & & $\because 1 \leq r$ & \\
\hline Seed size group & \multicolumn{3}{|c|}{ root dry weight $\left(\mathrm{g} \mathrm{pot}^{-1}\right)$} & $\begin{array}{l}\text { Seed size effect } \\
\text { Adj. LSD, } 0.05\end{array}$ & & \\
\hline $\mathrm{I}$ & \multicolumn{3}{|c|}{.190} & \multirow{3}{*}{$\because \cdot 1 r$} & & \\
\hline II & \multirow{2}{*}{\multicolumn{3}{|c|}{$\begin{array}{l}.170 \\
.111\end{array}$}} & & & \\
\hline III & & & & & & \\
\hline
\end{tabular}

Table (4): Effects of barley variety and seed size group(I, II and III) on shoot dry weight of seedling.

\begin{tabular}{|c|c|c|c|c|c|c|}
\hline \multirow[b]{2}{*}{ Barley varieties } & \multicolumn{3}{|c|}{ shoot dry weight $\left(\mathrm{g} \mathrm{pot}^{-1}\right)$} & \multirow{2}{*}{$\begin{array}{c}\text { Variety } \times \text { seed size effect } \\
\text { Adj. LSD }, 0.05\end{array}$} & \multirow{2}{*}{$\begin{array}{l}\text { Shoot dry } \\
\text { weight } \\
\left(\mathrm{g} \mathrm{pot}^{-1}\right)\end{array}$} & \multirow[b]{2}{*}{$\begin{array}{c}\text { Variety effect } \\
\text { Adj. LSD, } \\
0.05\end{array}$} \\
\hline & I & II & III & & & \\
\hline El-Kheir & 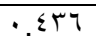 &.$r V V$ &. YVA & \multirow{15}{*}{$\because .91$} &. & \multirow{15}{*}{. .07} \\
\hline Buraq &.$\% 19$ & $\cdot r \cdot \varepsilon$ &. YYA & & $\cdot Y \wedge \varepsilon$ & \\
\hline Ghallion & 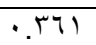 &. ro. & $\cdot r \leqslant Y$ & &.$\mu 1 \wedge$ & \\
\hline $1 / 1105$ & 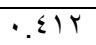 & $\cdot . \leqslant 11$ & $. Y Y \leq$ & & $\cdot r \leqslant q$ & \\
\hline Haddhar-465 & $\cdot r \leqslant r$ & $\cdot r \leqslant 0$ & $\cdot Y Y \leq$ & & $\cdot Y V I$ & \\
\hline Amal & . & $\cdot$ TrV &.$r 17$ & & 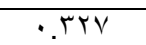 & \\
\hline Al-Bawadi & (וT) & $\cdot Y r V$ &. $.1 \vee \wedge$ & & $\cdot r \leq q$ & \\
\hline Sameer &.$\mu I r$ & $\cdot Y \wedge \uparrow$ &.$r O \Lambda$ & & $\cdot Y \wedge \uparrow$ & \\
\hline Local black & $\cdot \varepsilon \cdot r$ & $\cdot r \cdot \varepsilon$ & $\cdot Y I T$ & & $\cdot r \cdot \tau$ & \\
\hline Biba-99 & $\cdot . \mathrm{rqT}$ & $\cdot r q r$ &.$r \wedge T$ & & $\because r \wedge q$ & \\
\hline ACSAD-14 & $\cdot r 0 \Lambda$ & $\cdot Y \wedge$. & $\cdot r \leqslant Y$ & & $\cdot$. Yq & \\
\hline ACSAD-2 & $\cdot r \cdot \cdot$ & $\cdot . \wedge \wedge 9$ &. .18 & & . YOT & \\
\hline ACSAD-9 & . &. ro. & . YOT & &.$\mu \cdot 0$ & \\
\hline ACSAD-12 & $\because \varepsilon \cdots$ & $\cdot Y V \leq$ & $\cdot Y V \leq$ & & .117 & \\
\hline Najim Al-deen & . YY7 & $\cdot Y Y V$ & $\cdot Y \cdot \Sigma$ & & . YMY & \\
\hline Seed size group & \multicolumn{3}{|c|}{ Shoot dry weight $\left(\mathrm{g} \mathrm{pot}^{-1}\right)$} & \multirow{2}{*}{$\begin{array}{l}\text { Seed size effect } \\
\text { Adj. LSD, } 0.05\end{array}$} & & \\
\hline I & \multicolumn{3}{|c|}{$\cdot r \leqslant 7$} & & & \\
\hline II & \multicolumn{3}{|c|}{$\cdot r \cdot \varepsilon$} & \multirow[b]{2}{*}{$\because$ ro } & & \\
\hline III & \multicolumn{3}{|c|}{.$r M q$} & & & \\
\hline
\end{tabular}

Our results suggests that larger seed size and higher seed micronutrients content are very important for early seedling growth and might be overcome 
problems temporary of insufficient micronutrient content of alkaline calcareous soil.To our knowledge this is the first study and report of a large project in Iraq dealing with screening for micronutrients efficient and inefficient varieties of cereals and its adaptive strategies in calcareous soil.
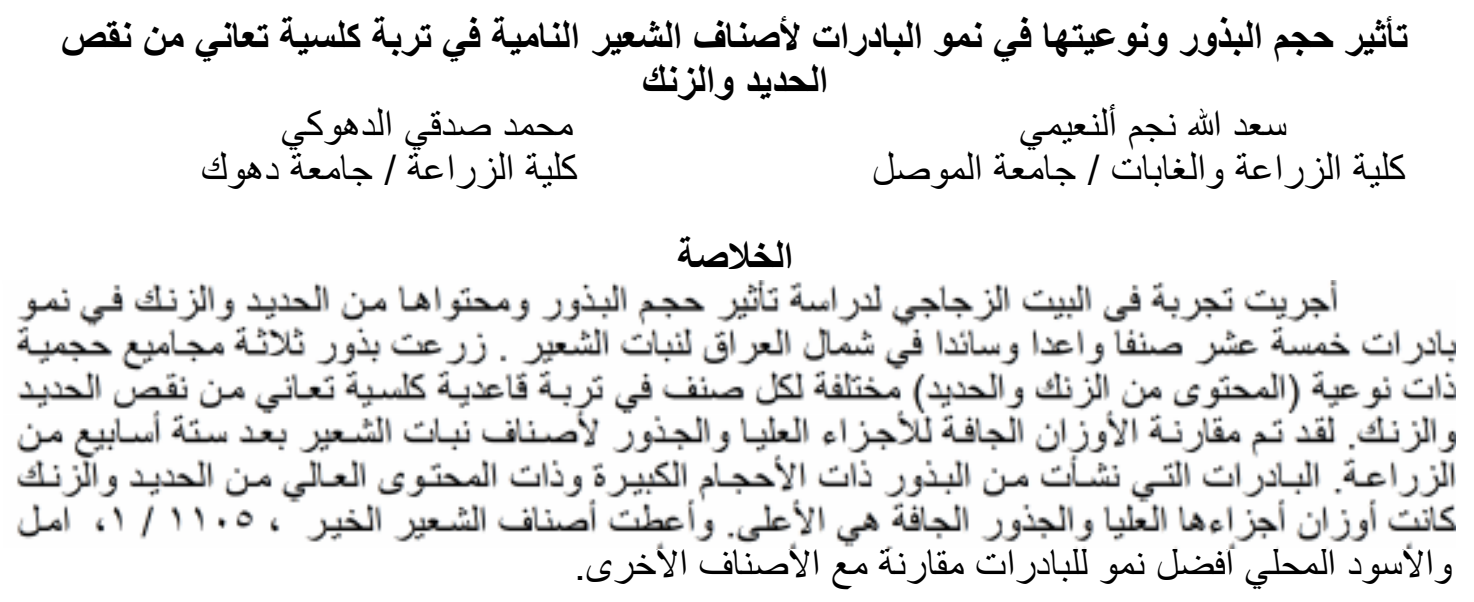

\section{REFERENCES}

Chapman, H. D. and P. E. Pratt (1961). Methods of Analysis for Soils, Plant and Water. Univ. of Calif., Davis, Division of Agric. Sci. P: 309.

Main, A. R. and E. D. Nafziger (1992). Seed size effects on emergence, head number, and grain yield of Winter wheat. J. Prod. Agric. 5: 265-268.

Marschner, H. (1995). Mineral Nutrition of Higher Plants. Academic Press London.

Mengel, K. and E. A. Kirkby (2001). Principles of Plant Nutrition. $5^{\text {th }}$ ed. Kluwer Academic publishers.

Moussavi-Nik, M.; Z. Rengel; G. J. Hollamby and J. S. Ascher (1997). Seed manganese $(\mathrm{Mn})$ content is more important than $\mathrm{Mn}$ fertilization for wheat growth under Mn- deficient conditions. In: Plant nutrition for sustainable food production and environment. T. Ando (eds.), pp. 267-268, Kluwer Academic publishers, Dordrecht.

Rengel, Z. and R. D. Graham (1995). Importance of seed Zn content for wheat growth on zinc-deficient Soil. I. Vegetative growth. Plant and Soil. 173: 259266. Sims, J. T. and G. V. Johnson (1991). Micronutrient soil tests, In: Micronutrient in Agriculture. $2^{\text {nd }}$ ed. Eds. Mordvedt J. J. et al., PP. 427-476. The Soil Science Society of America Book Series NO. 4, Madison, WI,. USA.

Soltanpour, P. N. and A. P. Schwab (1977). A new soil test for simultaneous extraction of Macro- and Micro- nutrient in alkaline soil. Communications in Soils Sci. and Plant Analysis. 8: 195-207.

Yilmaz, A.; H. Ekiz; I. Gultekin; B. Torun; S. Karanlik and I. Cakmak (1997). Effect of seed zinc content on grain yield and zinc concentration of wheat grown in zinc- deficient calcareous soils. In: Plant nutrition for sustainable food production and environment. T. Ando (eds.) pp. 283-284. Kluwer Academic publishers, Dordrecht. 